\title{
Numerical solving for optimal control of stochastic nonlinear system based on PF-PSO
}

\author{
Xiong Yong ${ }^{1,2^{*}}$, Xiaohao $\mathrm{Qu}^{1,2}$ \\ ${ }^{1}$ School of Navigation, Wuhan University of Technology, Wuhan 430063,Hubei, China \\ ${ }^{2}$ Hubei Inland Shipping Technology Key Laboratory, Wuhan 430063,Hubei, China \\ "bear_brave@163.com, 122127671@qq.com
}

\begin{abstract}
Keywords: optimal control; stochastic nonlinear; particle filtering; swarm optimization algorithm Abstract. Getting general solution for optimal control of stochastic nonlinear system is a hard task, the main difficulty is state variable and the control variable influencing each other,so system does not satisfy the separation theorem. To solve this problem, this paper use horizon optimal control strategy, adopting particle filtering method to get system state variable and variance function in every step with regard to measure value, then changing multi-variable function about control variable into optimizing index function, finally, using particle swarm optimization algorithm to solve global extreme value of this multimodal function, then, numerical solution for the problem was found. Simulated experiment result indicates that this method is an appropriate method for optimal control of stochastic nonlinear system and shows fairly good effect.
\end{abstract}

\section{Introduction}

The optimal control of stochastic system is an important research filed in automatic control. Many practical problems can be attributed to automatic control, such as vehicle vibration control, reservoir dispatch management 、 stock investment. However the general solution of optimal control of stochastic systems is very difficult to get. So far, more research focus on LQG problems, which is linear-quadratic-Gaussian control, the equation of state and measure equation of system are both linear model and the inputted random noise is additive Gaussian noise which is independent of each other, the optimizing index is the quadratic performance index. As these problems meet of the separation theorem, and the state estimation and optimal control can be calculated separately, So it can achieve online closed-loop optimal control at least in theory, these issues has introduced in many textbooks[1], we will no longer redundant.

However, it's more difficult to solve the stochastic optimal control of nonlinear systems. So far, there is no general solution for such questions. The existing methods are mainly for some certain specific types. The main difficulty can be ascribed to two factors: first, stochastic optimal control of nonlinear systems don't satisfy the separation theorem, so state estimation and optimal control can't be calculated separately, both influence each other, the input control variables both influence the performance index to be optimized and state estimation, so it's difficult to solve system state estimation by dynamic programming. Second, even if it can complete the state estimation, because the state equation or measure equation is nonlinear, the result of state estimates and the Bellman equation is also nonlinear through Bellman dynamic programming. It's difficult to get the analytic solutions of this equation in general, A more realistic approach is to seek the numerical solution in sub-optimal. The Bellman equation to be solved is multivariate nonlinear function, which contain many extreme points in general, it's difficult to deal with by any kinds of gradient methods because it is local optimization algorithms, unless global optimization algorithm is used. Due to the above reasons, the real-time state estimated and the Bellman equation must be solved separately for the control of stochastic nonlinear systems, however, these two issues are not separate for nonlinear stochastic system, but interact on each other, that is control variables affect both the state estimation and the index function to be optimized .we must find an effective method to solve these problems. 
At present, the solution of numerical method of stochastic nonlinear systems for optimal control are two main types: the stochastic control based on Markov chain and the perturbation control based on nominal state[2], The former's main idea is to divide the system variables and control variables into countable discrete values, and assume that these variables have the Markov nature and solve the Bellman equation iteration under the assumption, The latter's main idea is to expand the non-linear system equation with the polynomial, get the approximate value, then under the premise of the standard state equation to solve perturbation. As mentioned above, both two methods require the system to satisfy some assumptions, and will encounter "dimension disaster" problems, so the application has some limitations.

This paper proposes a common numerical solution to solve the problem which is based on a kind of rolling optimization control, combined the particle filtering with the particle swarm optimization algorithm. The main idea is using time-varying index function for optimize and particle filtering to realize state variable estimation which including incomplete measure information, then adopting dynamic programming to meet the Bellman equation, finally solving the numerical solution of this equation by particle swarm optimization algorithm. at last this paper gives a simulation example, analyze the results, points out the advantages and disadvantages of this method and puts forward the direction of further research.

\section{The Particle Filtering Method for Nonlinear Stochastic System.}

Particle Filtering (PF in short) methods used to deal with the state estimation problems of non-linear, non-Gaussian system[3]-[6], These methods primarily based on two thoughts which are sequence importance sampling and discrete approximation of probability density function[7]-[8], their differences are the selection of important function and the improvement of particles diversity. Consider the nonlinear stochastic system such as (1) and (2)

$$
\begin{aligned}
& x_{k+1}=f_{k}\left(x_{k}, w_{k}\right) \\
& y_{k}=h_{k}\left(x_{k}, v_{k}\right)
\end{aligned}
$$

$x_{k} \in R^{n}, y_{k} \in R^{m}, k \in N, v_{k}$ and $w_{k}$ are random variables for suitable dimension which is depends on the concrete form of equation (1) and (2). The marginal posterior distribution of $p\left(x_{k} \mid Y_{k}\right)$ about $x_{k}$, $Y_{k}=\left(y_{1}, y_{2}, \ldots y_{k}\right)$ was estimated if measure variable $y_{k}$ have got, $(k=1 \ldots n)$, and then we can get the estimated value $\hat{x}_{k}$ at step $k$, according to particle filtering methods, the following iteration formulas are:

$$
\begin{aligned}
& p\left(x_{k} \mid Y_{k}\right)=\sum_{i=1}^{N} w_{k}^{i} \delta\left(x-x_{k}^{i}\right) \\
& \hat{x}_{k}=\int x_{k} p\left(x_{k} \mid Y_{k}\right) d x_{k}=\int \sum_{i=1}^{N} w_{k}^{i} x_{k} \delta\left(x_{k}-x_{k}^{i}\right) d x_{k}=\sum_{i=1}^{N} w_{k}^{i} x_{k}^{i}
\end{aligned}
$$

Among them, $w_{k}^{i}$ is weight, iterative calculation as follows:

$$
w_{k}^{i}=w_{k-1}^{i} \frac{p\left(y_{k} \mid x_{k}\right) p\left(x_{k} \mid x_{k-1}^{i}\right)}{q\left(x_{k} \mid x_{k-1}^{i}, y_{k}\right)}
$$

According to the above iterative weighting function, the weight value is mainly affected by the important function $q\left(x_{k} \mid x_{k-1}^{i}, y_{k}\right)$, And when the important functions satisfy (6), the variance of weight function reaches a minimum. 


$$
q\left(x_{k} \mid x_{k-1}^{i}, y_{k}\right)_{o p t}=p\left(x_{k} \mid x_{k-1}^{i}, y_{k}\right)=\frac{p\left(y_{k} \mid x_{k}, x_{k-1}^{i}\right) p\left(x_{k} \mid x_{k-1}^{i}\right)}{p\left(y_{k} \mid x_{k-1}^{i}\right)}
$$

Substitute (6) into (5):

$w_{k}^{i}=w_{k-1}^{i} p\left(y_{k} \mid x_{k-1}^{i}\right)$

Assume that in step $k$, known $x_{k-1}^{i}$ and $w_{k-1}^{i}, i=1,2 \ldots m$, The basic steps for particle filtering are:

1. According to the (1), update sample value $x_{k-1}^{i}, x_{k}^{i}=f_{k}\left(x_{k-1}^{i}, w_{k-1}^{i}\right)$, record the new measure variable ${ }^{y_{k}}$.

2. According to (7), update weight value $w_{k-1}^{i}, w_{k}^{i}=w_{k-1}^{i} p\left(y_{k} \mid x_{k-1}^{i}\right)$.

3. According to (4), obtain the new estimated value $\hat{x}_{k}$ in step $k$.

4. Iterative next step, go to step 1.

\section{Particle Swarm Optimization Algorithm}

Particle Swarm Optimization (PSO in short) algorithm is a random, parallel global optimization algorithm. Kennedy and Eberhart absorbed the enlightenment form the foraging behavior of animal groups, found that the groups in the search for a target, for some individual, it is often refer to the individual which in the best position of the group currently and the individual itself once achieved the best position, to adjust the next search direction and velocity, thus the particle swarm optimization algorithm is designed. PSO can be used to calculate the global extreme point of complex nonlinear multimodal function, the basic PSO iteration formulas are [9]-[11] :

$$
\begin{aligned}
& v_{i d}(t+1)=v_{i d}(t)+c_{1} \varphi_{1}\left(p_{i d}(t)-x_{i d}(t)\right)+c_{2} \varphi_{2}\left(p_{g d}(t)-x_{i d}(t)\right) \\
& x_{i d}(t+1)=x_{i d}(t)+v_{i d}(t)
\end{aligned}
$$

Subscript $i$ and $d$ respectively are particle number and number of dimensions, $v_{i d}$ and $x_{i d}$ respectively express the speed and position of each particle, $t$ is the step number of iterative computation, $c_{1}$ and $c_{2}$ are the system controlled variables, $\varphi_{1}, \varphi_{2}$ are random numbers in $(0,1)$ interval, $p_{i d}$ is the best optimized results which particle $i$ obtains until now, $p_{g d}$ is the position of particle which could obtain the best optimization results in all groups so far. The basic steps for PSO solving problem are:

1 Initialize the positions and velocities of particles.

2 Calculated the fitness value for each particle and the global best value for all the particles.

3 Update the position and speed of particles by formula (8) and (9).

4 Test Pre-set conditions about ending compute criterion whether is satisfied, if not, return to 2.

\section{Estimate the state of stochastic nonlinear control systems by particle filtering}

If Particle filtering be used for stochastic nonlinear systems under the quadratic optimal control, firstly, the particle filtering must be expanded to a more general situation about control variables. Such as types (10) and (11) shows, $x_{k} \in R^{n}, y_{k} \in R^{m}, u_{k} \in R^{n}, k \in N, v_{k} \in R^{m}, k \in N$ satisfy the normal distribution, ${ }^{w_{k}}$ is the random variable for the corresponding dimension.

$$
\begin{aligned}
& x_{k+1}=f_{k}\left(x_{k}, w_{k}\right)+\Gamma u_{k} \\
& y_{k}=h_{k}\left(x_{k}\right)+v_{k}
\end{aligned}
$$


For the stochastic nonlinear systems like form (10) and (11), the initial value of state variables $x_{0}^{i}$ and measure variables $y_{k}$ is known, $k=1 \ldots n$, so optimal control problem as follows : $\min J_{m}(k)=E\left\{W\left(\Psi_{k}, m\right)\right\}$

$$
W\left(\Psi_{k}, m\right)=\min _{u(k), \ldots, u(k+m-1)} E\left\{\sum_{i=k}^{k+m-1}\left[x^{T}(i) Q_{1} x(i)+u^{T}(i) Q_{2} u(i)\right] \psi_{k}\right\}
$$

With $\psi_{k}=\left\{\mathbf{y}^{k}, \mathbf{u}^{k-1}\right\}$

$$
\begin{aligned}
& \mathbf{y}^{k}=\{y(1), y(2), \ldots y(\mathrm{k})\} \\
& \mathbf{u}^{k-1}=\{u(1), u(2), \ldots u(\mathrm{k}-1)\}
\end{aligned}
$$

The Bellman dynamic programming equation is:

$W\left(\Psi_{k}, m\right)=\min _{u(k)} E\left\{x^{T}(k) Q_{1} x(k)+u^{T}(k) Q_{2} u(k)+W\left(\Psi_{k+1}, m\right) \mid \psi_{k}\right\}$

Because: $E\left\{x^{T}(k) Q_{1} x(k) \mid \psi_{k}\right\}=x^{T}(k \mid k) Q_{1} x(k \mid k)+\operatorname{tr} Q_{1} P(k \mid k)$

So:

$$
W\left(\Psi_{k}, m\right)=\min _{u(k)}\left\{x^{T}(k \mid k) Q_{1} x(k \mid k)+\operatorname{tr} Q_{1} P(k \mid k)+u^{T}(k) Q_{2} u(k)+W\left(\Psi_{k+1}, m\right)\right\}
$$

Using equation (17) recursive Calculates until to $k+m-1$ step, so:

$$
W\left(\Psi_{k}, m\right)=\min _{u(k), \ldots, u(k+m-1)} \sum_{l=k}^{k+m-1}\left(x^{T}(l \mid l) Q_{1} x(l \mid l)+\operatorname{tr} Q_{1} P(l \mid l)+u^{T}(l) Q_{2} u(l)\right)
$$

Among them, $x(k \mid k)$ is the estimate value at step $k, P(k \mid k)$ is the variance of estimate error at step $k$, It is no longer meet separation theorem in nonlinear circumstances, so $P(k \mid k)$ is a function of control variable $u(k-1)$, and $\operatorname{tr} Q_{1} P(k \mid k)$ is the trace of matrix $Q_{1} P(k \mid k)$, so:

$$
x(k \mid k)=\sum_{i=1}^{N} w_{k}^{i} x_{k}^{i}=\sum_{i=1}^{N} w_{k-1}^{i} p\left(y_{k} \mid x_{k-1}^{i}\right) x_{k}^{i}
$$

In order to obtain the posterior distribution $p\left(x_{k} \mid Y_{k}\right)$, the most important is to get the value of $p\left(y_{k} \mid x_{k-1}^{i}\right)$, so the extend kalman filtering is adopted to resolve this problem by using linear approximate method.

Assume that sampling $i$ particles at the same time, and each particle meet Gaussian distribution and linear approximation, $\operatorname{note}\left(x_{k}^{i}, P_{k}^{i}\right)_{i=1,2 \ldots \ldots N}, P_{k}^{i}$ is variance.

$$
\begin{aligned}
& P_{k}^{i}=E\left[\left(x_{k}-x_{k}^{i}\right)\left(x_{k}-x_{k}^{i}\right)^{T}\right] \\
& x_{k \mid k-1}^{i}=f_{k}\left(x_{k-1}^{i}, w_{k-1}^{i}\right)+\Gamma u_{k-1} \\
& P_{k \mid k-1}^{i}=F_{k-1}^{i} P_{k-1}^{i} F_{k-1}^{i^{T}}
\end{aligned}
$$

$F_{k}^{i}$ is the Jacobian matrix of $f_{k}\left(x_{k}\right)$.

$x_{k}^{i}=x_{k \mid k-1}^{i}+K_{k}^{i}\left(y_{k}-h_{k}\left(x_{k \mid k-1}^{i}\right)\right)+\Gamma u_{k-1}^{i}$

$K_{k}^{i}=P_{k \mid k-1}^{i} H_{k}^{i^{T}}\left(H_{k}^{i} P_{k \mid k-1}^{i} H_{k}^{i^{T}}+R_{k}\right)^{-1}$

$H_{k}^{i}$ is the Jacobian matrix of $h_{k}\left(x_{k}\right)$.

$$
P_{k}^{i}=P_{k \mid k-1}^{i}-K_{k}^{i} H_{k}^{i} P_{k \mid k-1}^{i}
$$

So:

$$
p\left(y_{k} \mid x_{k-1}^{i}\right)=N_{y_{k}}\left(h_{k}\left(x_{k \mid k-1}^{i}\right), H_{k}^{i} P_{k \mid k-1}^{i} H_{k}^{i^{T}}+R_{k}\right)
$$


$N_{y}(\mu, \sigma)$ Expresses the normal distribution density function of variable ${ }^{y}, \mu$ and $\sigma$ are the mean and variance respectively, then the weight is normalized .

$$
w_{k}^{i}=\frac{w_{k-1}^{i} N_{y_{k}}\left(h_{k}\left(x_{k \mid k-1}^{i}\right), H_{k}^{i} P_{k \mid k-1}^{i} H_{k}^{i^{T}}+R_{k}\right)}{\sum_{j=1}^{N} w_{k-1}^{i} N_{y_{k}}\left(h_{k}\left(x_{k \mid k-1}^{i}\right), H_{k}^{i} P_{k \mid k-1}^{i} H_{k}^{i^{T}}+R_{k}\right)}
$$

So the finally iterative formulas as follows:

$$
\begin{aligned}
& x(k \mid k)=\sum_{i=1}^{N} w_{k}^{i} x_{k}^{i}=\sum_{i=1}^{N} \frac{w_{k-1}^{j} N_{y_{k}}\left(h_{k}\left(x_{k \mid k-1}^{i}\right), H_{k}^{i} P_{k \mid k-1}^{i} H_{k}^{i}+R_{k}\right)}{\sum_{j=1}^{j} w_{k-1} N_{y_{k}}\left(h_{k}\left(x_{k \mid k-1}^{j}\right), H_{k}^{j} P_{k \mid k-1}^{j} H_{k}^{j^{T}}+R_{k}\right)} x_{k}^{i}=\sum_{i=1}^{N} \frac{w_{k-1}^{j} N_{y_{k}}\left(h_{k}\left(f_{k}\left(x_{k-1}^{i}, w_{k-1}^{j}\right)+\Gamma u_{k-1}\right), H_{k}^{i} P_{k \mid k-1}^{i} H_{y_{k}}\left(h_{k}\left(f_{k}\left(x_{k-1}^{j}, w_{k-1}^{j}\right)+\Gamma u_{k-1}\right), H_{k}^{j} P_{k \mid k-1}^{j} H_{k}^{j^{T}}+R_{k}\right)\right.}{\times\left\{f_{k}\left(x_{k-1}^{i}, w_{k-1}^{i}\right)+2 \Gamma u_{k-1}+K_{k}^{i}\left(y_{k}-h_{k}\left(f_{k}\left(x_{k-1}^{i}, w_{k-1}^{i}\right)+\Gamma u_{k-1}\right)\right)\right\}}
\end{aligned}
$$

This formula describes the recursive estimate value $x(k \mid k)$ by the particle filtering algorithm when $w_{k-1}^{i}$ and $x_{k-1}^{i}$ is known, therefore, $x(k \mid k)$ is the function of $w_{k-1}^{i} 、 x_{k-1}^{i}$ and $u_{k-1}$.

Using the following formula and formulas (21) and (24), and the expression of $w_{k}^{i}$ and $x_{k}^{i}$ about $w_{k-1}^{i}$ and $x_{k-1}^{i}$, then the $P(k \mid k)$ can be expressed as the function of $w_{k-1}^{i} 、 x_{k-1}^{i}$ and $u_{k-1}$.

$$
P(k \mid k)=\sum_{i=1}^{N} w_{k}^{i}\left[\left(x_{k}^{i}-\hat{x}_{k}\right)\left(x_{k}^{i}-\hat{x}_{k}\right)^{T}+P_{k}^{i}\right]
$$

\section{Using particle swarm optimization algorithm for solving the control variables}

According to the fore-mentioned, the state variable $x(k \mid k)$ and variance $P(k \mid k)$ are both the functions of $w_{k-1}^{i} 、 x_{k-1}^{i}$ and $u_{k-1}$ when $m=1$, and the optimizing objective function of the system is:

$$
W\left(\Psi_{k}, 1\right)=\min _{u(k)} \sum_{l=k}^{k}\left(x^{T}(l \mid l) Q_{1} x(l \mid l)+\operatorname{tr} Q_{1} P(l \mid l)+u^{T}(l) Q_{2} u(l)\right)
$$

Among them, $x(k \mid k)$ and $P(k \mid k)$ are decided by functions (28) and (29) respectively, so the objective function $W\left(\Psi_{k}, 1\right)$ is the nonlinear function of $w_{k-1}^{i} 、 x_{k-1}^{i} 、 u_{k-1}$ and Observed variable $y_{k}$, these four variables is known at the step $k$, and the numerical solution of $u(k)$ Can be obtained by Particle Swarm Optimization algorithm. The optimizing objective function can be expressed as follows when $m=r$ and $r \in N$ :

$$
W\left(\Psi_{k}, m\right)=\min _{u(k), u(k+1) \ldots, u(k+m-1)} \sum_{l=k}^{k+m-1}\left(x^{T}(l \mid l) Q_{1} x(l \mid l)+\operatorname{tr} Q_{1} P(l \mid l)+u^{T}(l) Q_{2} u(l)\right)
$$

Using the state equations (10) and the weight function expression (27), the $x(l \mid l)$ and $P(l \mid l)$ can be expressed as a function of $x_{k-1}^{i} 、 w_{k-1}^{i}$ and $y_{k}, \ldots y_{k+1-m} 、 u_{k}, \ldots u_{k+1-m}$, and then substitute it for function (15), therefore, $W\left(\Psi_{k}, m\right)$ also can be expressed as a function of $x_{k-1}^{i}$ 、 $w_{k-1}^{i}$ and $y_{k}, \ldots y_{k+1-m} 、 u_{k}, \ldots u_{k+1-m}$, and $x_{k-1}^{i} 、 w_{k-1}^{i}$ and measure value $y_{k}, \ldots y_{k+1-m}$ is known at step $k$, The problem is transformed into how to solve a Optimal value of multi-modal function $W\left(\Psi_{k}, m\right)$ about $u_{k}, \ldots u_{k+1-m}$, because this is multi-modal function which including both nonlinear and high-dimension, so it' $s$ difficult to be solved by the general optimization method, Therefore, we use PSO algorithm to solve the numerical solution of function (31). 
In summary, the solution of rolling optimization control of nonlinear stochastic optimization control system which is based on the PF-PSO method is as follows:

1. Initialization: set the initial parameters of the index steps $m$, particle filtering and particle swarm optimization algorithm when $i=k$.

2. State estimation: $\psi_{k-1}$ can be obtained by the previous steps, using particle filtering can calculate the estimated value of state variables from step $k$ to step $k+m-1$.

3. Solve the control variables: Using state estimation expressions, lists random Bellman equation, and then the numerical solution $u_{k}, \ldots u_{k+1-m}$ of objective function to be optimized can be solved by particle swarm optimization algorithm。

4. Iteration: $i=k+1$, Return to step 1 .

\section{Simulation}

Formulas (32) and (33) express an economic system, unsteady growth of single-variable models in the stochastic nonlinear system, solving the optimal control problem of formula (34) under the four-step quadratic index function. First, transform the quadratic function into multi-variant function $W\left(\Psi_{k}, 3\right)$ about unknown variable $u_{k}, \ldots u_{k+3}$, Then the global optimal of this function can be solved by the PSO algorithm .

$$
\begin{aligned}
& x=0.5 x+25 x /\left(1+x^{2}\right)+8 \cos (1.2 t)+Q \\
& y=x^{2} / 20+R
\end{aligned}
$$

$\mathrm{Q}$ and $\mathrm{R}$ is random variable with variance 1 , The initial value of the particle filtering is $x_{0}^{i}=1$, $i=1,2 \ldots 20, v_{k}$ and $w_{k}$ satisfy the normal distribution with mean 0 and variance is $R_{k}$, the objective function to be optimized is:

$$
J=\min _{u(k), \ldots, u(k+3)} E\left\{\sum_{i=k}^{k+3}\left[3 x^{2}(i)+4 u^{2}(i)\right] \mid Y_{k-1}\right\}
$$

Substitute the function of $P(k \mid k)$ into (34) according (29), the expression of index function (35) is available.

$$
W\left(\Psi_{k}, 3\right)=\min _{u(k), \ldots u(k+3)} \sum_{l=k}^{k+3}\left(3 x^{T}(l \mid l)^{2}+3 P(l \mid l)+4 u^{T}(l)^{2}\right)
$$

This function contains the initiative value $x_{k-1}^{i}$ and measure value $y_{k}, \ldots y_{k+3}$, and four control variables $u_{k}, \ldots u_{k+3}$ which are unknown; PSO algorithm is used to solve the global minimum point of multivariate nonlinear function (35). There are set 20 particles, the initial value of each particle's position and velocity are set as the unit vector[11...1]. Figure 1 is the evolution graphics of the estimated state variables by PF; Figure 2 is the evolution graphics of index function optimization by PSO. 


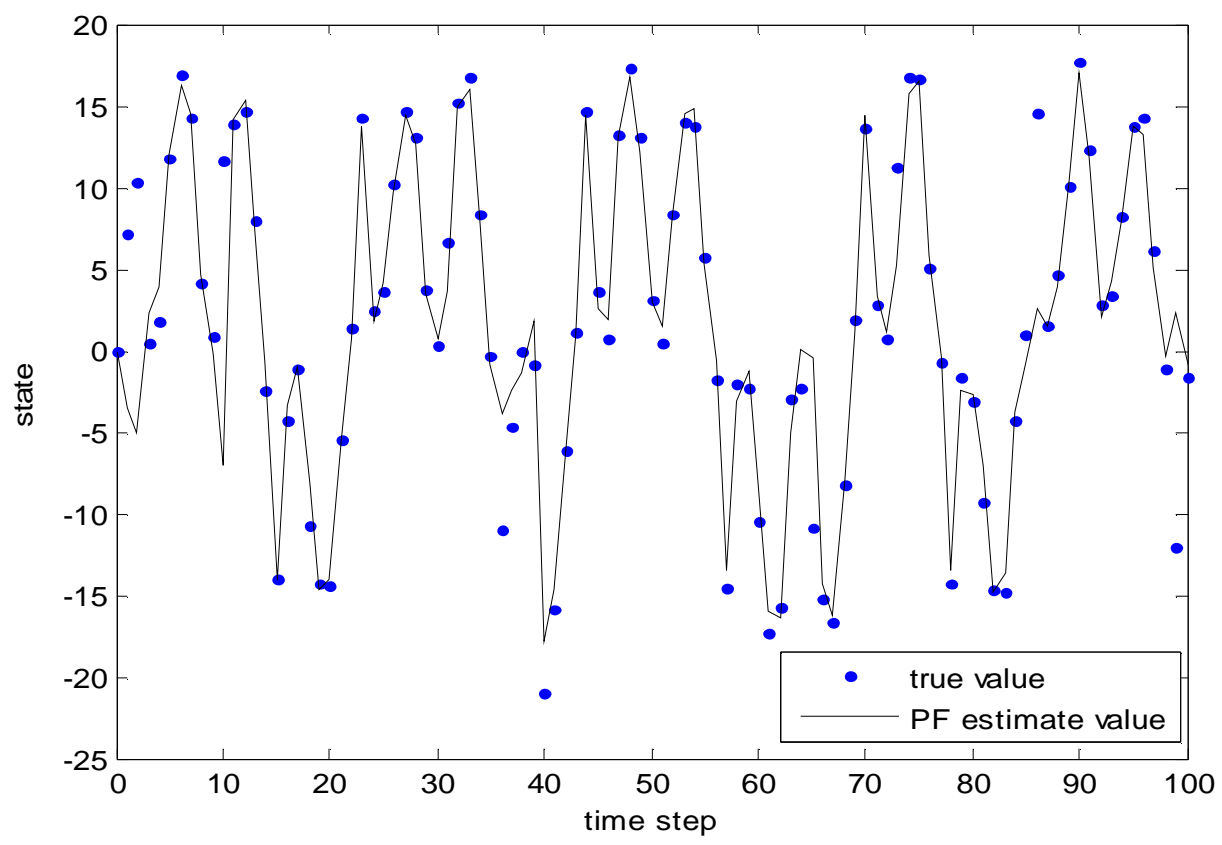

Figure 1 The curve of the estimated state value and the true value with the change of iterations

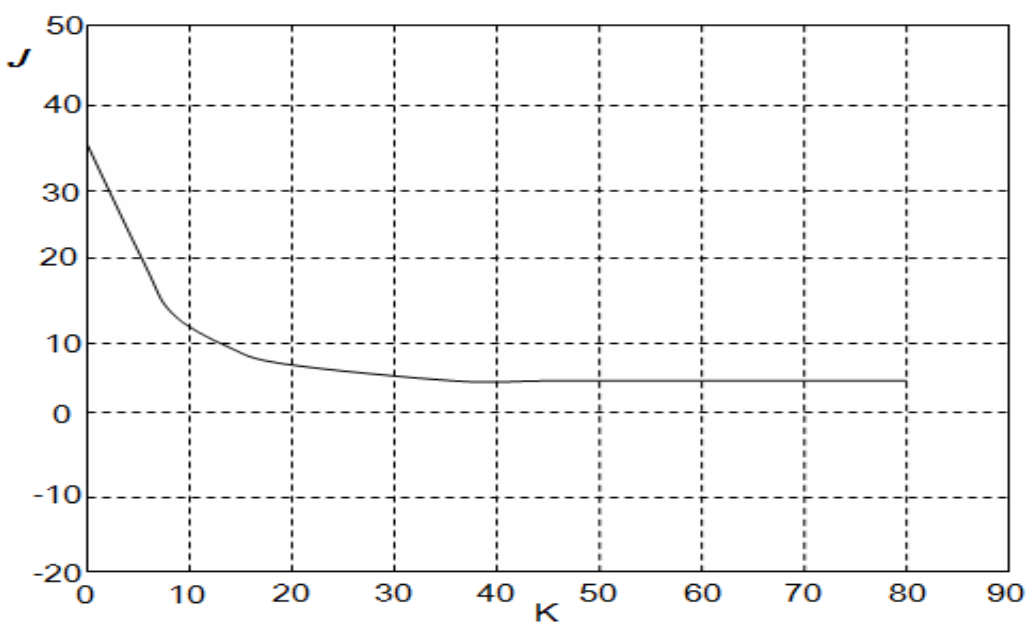

Figure 2 The curve of evolution graphics of index function optimization

The above two figures shows that, the accurate estimated value of state variables can be obtained after 60 70 steps iteration, while the stable optimization of indicator function can be obtained until 40 steps iteration. The weakness of this approach is great computation consume, because every step must solve the two calculation circle including filtering and optimization, and both algorithms require multi-particles parallel computing.

\section{Conclusion}

This paper presents a general framework for calculating such problems. In actual calculation, we can use appropriate improved algorithms according to specific state equation and measure equation, to improve the efficiency of the process and achieve better effect of calculation. From the practical point of view, how to improve the computational efficiency of this method is the next problem to be researched.

\section{Acknowledgments}

This 


\section{References}

[1] Guo S L. Stochastic control. Beijing: Tsinghua University Press, 1999

[2] Chang, Mou-Hsiung. Stochastic Control of Hereditary Systems and Applications.Springer-Verlag New York Inc, 2008

[3] Gordon N J, Salmond D J, Smith A F M. Novel approach to nonlinear/non-Gaussian Bayesian state estimation. IEE Proceedings-F, 1993, 140(2):107-113.

[4] Liu J S, Chen R. Sequential Monte Carlo methods for dynamic systems. J of the American Statistical Association, 1998, 93(443):1032-1044.

[5] Doucet A, Godsill S, Andrieu C. On sequential Monte Carlo sampling methods for Bayesian filtering. Statistics and Computing, 2000, 10(1):197-208.

[6] Doucet A, de Freitas J F G, Gordon N J. Sequential Monte Carlo Methods in Practice. New York: Springer-Verlag, 2001.

[7] Isard M, Blake A. Condensation-conditional density propagation for visual tracking. Int $\mathbf{J}$ of Computer Vision, 1998, 29(1):5-28

[8] Kanazawa K, Koller D, Russell S J. Stochastic simulation algorithms for dynamic probabilistic networks. Proc of the 11th Annual Conference on Uncertainty in AI. Canada: Morgan Kaufmann Publishers, 1995:346-351

[9] Kennedy J. and Eberhart R C. Particle swarm optimization. Proceedings of IEEE International Conference on Neural Networks, Piscataway, NJ,1995:1942-1948

[10] Kennedy J. The particle swarm: social adaptation of knowledge. Proceedings of IEEE International Conference on Evolutionary Computation, Indianapolis, 1997: 303-308

[11] Clerc M, Kennedy J. The particle swarm-explosion, stability, and convergence in a multidimensional complex space. IEEE Transactions on Evolutionary Computation, 2002, 6(1): 58 $-73$ 